\title{
Construction and Validation of Brain MRI Templates from a Korean Normal Elderly Population
}

\author{
Hyunna Lee1, Byung II Yoo², Ji Won Han², Jung Jae Lee³, San Yeo Wool Oh², \\ Eun Young Lee ${ }^{3}$, Jae Hyoung $\mathrm{Kim}^{4}$, and Ki Woong Kim ${ }^{1,2,5} \bowtie$ \\ ${ }^{1}$ Department of Brain and Cognitive Sciences, Seoul National University College of Natural Sciences, Seoul, Republic of Korea \\ ${ }^{2}$ Department of Neuropsychiatry, Seoul National University Bundang Hospital, Seongnam, Republic of Korea \\ ${ }^{3}$ Department of Psychiatry, Dankook University Medical College, Cheonan, Republic of Korea \\ ${ }^{4}$ Department of Radiology, Seoul National University Bundang Hospital, Seongnam, Republic of Korea \\ ${ }^{5}$ Department of Psychiatry, Seoul National University College of Medicine, Seoul, Republic of Korea
}

\begin{abstract}
Objective This study aimed to construct a Korean normal elderly brain template (KNE96) using Korean elderly individuals for use in brain MRI studies and to validate it.

Methods We used high-resolution 3.0T T1 structural MR images from 96 Korean normal elderly individuals (M/F=48/48), aged 60 years or older $(\mathrm{M}=69.5 \pm 6.2$ years, $\mathrm{F}=70.1 \pm 7.0$ years), for constructing the KNE96 template. The KNE96 template was validated by comparing the registration-induced deformations between the KNE96 and ICBM152 templates using different MR images from 48 Korean normal elderly individuals $(\mathrm{M} / \mathrm{F}=24 / 24)$, aged 60 years or older $(\mathrm{M}=71.5 \pm 5.9$ years, $\mathrm{F}=72.8 \pm 5.1$ years). We used the magnitude of displacement vectors (mag-displacement) and log of Jacobian determinants (log-Jacobian) to quantify the deformation produced during registration process to templates.

Results The mag-displacement and log-Jacobian of the registration were much smaller using the KNE96 template than with the ICBM152 template in most brain regions. There was a prominent difference in the significant averaged differences (SADs) of the magdisplacement and log-Jacobian between the KNE96 and ICBM152 at the superior, medial, and middle frontal gyrus, the lingual, inferior, middle, and superior occipital gyrus, and the caudate and thalamus.

Conclusion This study suggests that templates constructed from Asian populations, such as the KNE96, may be more desirable than those from Caucasian populations, like the ICBM152, in computational neuroimaging studies that measure and compare anatomical features of the frontal and occipital lobe, thalamus and caudate.

Psychiatry Investig 2016;13(1):135-145
\end{abstract}

Key Words Magnetic resonance imaging, Brain template, Korean, Elderly.

\section{INTRODUCTION}

Following advances in human brain imaging technologies, there have been several diverse studies using high quality brain magnetic resonance imaging (MRI) in both research and clinical fields. ${ }^{1-4}$ In many studies comparing brain MR images between different groups, a standard brain atlas or template is necessary to objectively localize and compare specific target

Received: January 7, 2015 Revised: May 21, 2015

Accepted: June 3, 2015 Available online: October 27, 2015

$\triangle$ Correspondence: Ki Woong Kim, MD, PhD

Department of Psychiatry, Seoul National University Bundang Hospital, Seoul National University College of Medicine, 82 Gumi-ro 82beon-gil, Bundanggu, Seongnam 13620, Republic of Korea

Tel: +82-31-787-7432, Fax: +82-31-787-4058, E-mail: kwkimmd@snu.ac.kr

(a) This is an Open Access article distributed under the terms of the Creative Commons Attribution Non-Commercial License (http://creativecommons.org/licenses/by$\mathrm{nc} / 3.0$ ) which permits unrestricted non-commercial use, distribution, and reproduction in any medium, provided the original work is properly cited. regions in individual brains because of differences in shape, size, and relative orientation. A brain template provides a standard anatomical reference for individual- or population-based assessments of brain structure and function. Spatial normalization to the brain template, which serves as a common coordinate system to control for anatomical differences in individual brains, is an important processing step in computational brain MRI analysis. ${ }^{5-9}$

A commonly used human brain coordinate system was initially presented by Talairach and Tournoux. ${ }^{10,11}$ The Talairach atlas was created from the postmortem sections of a 60 -yearold French woman; therefore, it was often criticized for being biased towards the subject of choice and lacking group-representative anatomical features. The International Consortium for Brain Mapping (ICBM) created another standard brain template to address the limitations of the Talairach atlas by 
averaging a large group of MRI scans from 305 young normal subjects $(\mathrm{M} / \mathrm{F}=239 / 66,23.4 \pm 4$.1 years). These scans were first spatially normalized to the Talairach space by linear registration and then averaged. ${ }^{5,9}$ One of the most popular brain templates is the ICBM152 atlas, which was created by averaging 152 normal MRI scans aligned into a common space using a 9-parameter transformation. ${ }^{12-14}$ Recently, a nonlinear version of the ICBM atlas has been introduced. ${ }^{15,16}$ The ICBM templates are adopted by many groups and incorporated in various MR image analysis tools, such as Statistical Parametric Mapping (SPM, Institute of Neurology, University College of London, UK), ${ }^{17}$ MriStudio, ${ }^{18}$ and FMRIB Software Library (FSL, University of Oxford, UK). ${ }^{19,20}$

However, these brain templates do not account for differences across phenotypic groups (e.g., age, gender, race, or disease conditions). ${ }^{21-25}$ The use of brain atlases in spatial normalization is typically limited to studies involving subject cohorts of similar phenotype; therefore, new population-specific brain templates have been created and recommended for use with other cohorts that are substantially different from the populations that were used to generate the existent templates. ${ }^{24-26}$ It has been demonstrated that Oriental and Occidental populations are dissimilar due to environmental and genetic influences. ${ }^{27,28}$ Thus, any deviation between the Caucasian brain template and subjects from Oriental cohorts leads to erroneous results in anatomical localization. Consequently, this can lessen the accuracy and power of any statistical analysis of the spatially normalized images.

For this reason, several efforts have been made to create an Asian population template, taking into account racial differences in brain shape and size, which can be used to perform spatial normalization processing. Lee et al. ${ }^{29}$ have constructed a Korean brain template using MRI and Positron-Emission Tomography (PET) images of 78 normal right-handed Koreans aged between 18 to 77 years $(\mathrm{M} / \mathrm{F}=49 / 29,44.6 \pm 19.4$ years). Tang et al. ${ }^{30}$ have created a Chinese brain template from 56 right-handed Chinese young male subjects $(24.46 \pm 1.81$ years). Sato et al. ${ }^{31}$ have developed a database of brain images from 1547 healthy Japanese subjects, aged 16 to 79 years, and Uchiyama et al. ${ }^{32}$ have created a brain template of 180 Japanese children $(\mathrm{M} / \mathrm{F}=75 / 105)$ between the ages of 5 and 9 years. However, to the best of our knowledge, no previous study has quantitatively validated these Asian templates by comparison with the ICBM templates, in terms of the registration-induced deformation.

In this study, we constructed a brain MRI template from Korean normal elderly subjects that would be fundamental for brain MRI studies about aging and geriatric. Specifically, we constructed a Korean normal elderly template (i.e., KNE96 template) using 3.0T T1 structural MRI images from 96 Ko- rean elderly subjects. Further, we validated the KNE96 template by comparing with the ICBM152 template, which is one of most popular templates based on a Caucasian population. Using 48 Korean elderly subjects, we validated the KNE96 template by comparing the deformation produced by image registration to the KNE96 template with that to the ICBM152 template.

\section{METHODS}

\section{Study population}

144 righted-handed Korean elderly subjects with normal cognition were selected from participants of the Korean Longitudinal Study on Cognitive Aging and Dementia (KLOSCAD), which is a population-based prospective elderly cohort study on cognitive aging and dementia that was launched in 2009. ${ }^{33}$ Participants of KLOSCAD were drawn from individuals aged 60 years or older. All participants assessed on clinical grounds to be cognitively normal in accordance with a Clinical Dementia Rating (CDR) ${ }^{34}$ scale of 0. Participants who had Axis I disorders listed in the DSM-IV ${ }^{35}$ or any serious medical or neurological disorders that could affect their cognitive function were excluded. Each subject was evaluated using the Korean version of the Consortium to Establish a Registry for Alzheimer's Disease (CERAD-K) ${ }^{36}$ by a research geropsychiatrist with expertise in dementia research. The Edinburgh handedness inventory was used to assess handedness. ${ }^{37}$ According to these criteria, we selected 144 rightedhanded Korean elderly subjects with normal cognition for this study. This study protocol was approved by the Institutional Review Board of Seoul National University Bundang Hospital (SNUBH). All subjects were fully informed about the study protocol. All subjects provided written informed consents that were signed by the subjects.

The 144 subjects were divided into two groups. 96 subjects $(\mathrm{M} / \mathrm{F}=48 / 48)$ aged 60 years or older $(\mathrm{M}=69.5 \pm 6.2$ years, $\mathrm{F}=$ $70.1 \pm 7.0$ years) were used to construct a Korean normal elderly template (i.e., KNE96 template), and the remaining 48 subjects $(\mathrm{M} / \mathrm{F}=24 / 24)$ aged 60 years of older $(\mathrm{M}=71.5 \pm 5.9$ years, $\mathrm{F}=72.8 \pm 5.1$ years) were used to validate the KNE96

Table 1. Sex and age distribution of the subjects used in this study

\begin{tabular}{ccccccc}
\hline \multirow{2}{*}{ Age } & \multicolumn{2}{c}{ Construction group } & & \multicolumn{2}{c}{ Validation group } \\
\cline { 2 - 3 } \cline { 5 - 6 } & Male & Female & & Male & Female \\
\hline $60-64$ & 12 & 12 & & 1 & 0 \\
$65-69$ & 12 & 12 & & 11 & 7 \\
$70-74$ & 12 & 12 & & 7 & 10 \\
$75-$ & 12 & 12 & & 5 & 7 \\
Total & 48 & 48 & & 24 & 24 \\
\hline
\end{tabular}


template. The sex and age distribution of the two groups of subjects is shown in Table 1 .

\section{Image acquisition and preparation}

Three-dimensional (3D) T1-weighted spoiled gradient echo (SPGR) MRI images were acquired from all subjects on a 3.0Tesla GE SIGNA scanner (GE Medical Systems, Milwaukee, USA). The imaging parameters were as follows: $1.0 \mathrm{~mm}$ sagital slices, echo time (TE) of $3.68 \mathrm{~ms}$, repetition time (TR) of $25.0 \mathrm{~ms}$, flip angle of $30^{\circ}$, and field of view (FOV) of $24 \times 24$ $\mathrm{cm}$. The acquisition matrix was $175 \times 240 \times 240$ in the $x-, y-$, and $\mathrm{z}$-dimensions yielding a voxel size of $1.0 \times 1.0 \times 1.0 \mathrm{~mm}^{3}$.

All data were acquired in DICOM format and then imported into ANALYZE format for analysis using the import function in SPM8. Each MRI scan was reoriented to virtual $\mathrm{x}-\mathrm{y}-\mathrm{z}$ coordinates so that the anterior commissural-posterior commissural (AC-PC) line of the brain was matched to the $\mathrm{y}$-axis, the vertical anterior commissural (VAC) line to the $\mathrm{z}$ axis, and the midsagittal plane to the $y-z$ plane.

\section{Global brain features}

Global brain shape and size are useful baselines for characterizing and comparing individual brains, although they do not provide detailed morphometry. Length, width, height, and volume of the whole brain are used as representative features of global brain shape and size. We measured the global brain features of each brain of 96 subjects in the construction group. We manually specified six reference points using BrainSuite: ${ }^{38,39}$ the most anterior (AP), posterior (PP), right (RP), left (LP), superior (SP), and inferior (IP) points of the cerebrum. The length, width, and height of the whole brain were determined as the distances between AP and PP, RP and LP, and SP and IP, respectively. Following this, the width to length (W/L), height to length $(\mathrm{H} / \mathrm{L})$, and height to width $(\mathrm{H} / \mathrm{W}) \mathrm{ra}-$ tios were computed. We automatically extracted tissue probability maps of white matter (WM), grey matter (GM), and cerebrospinal fluid (CSF) using the segmentation routine in SPM $8 .^{40}$ Whole brain volume was determined as the number of $1.0 \mathrm{~mm}^{3}$ isovoxels within which the sum of the three tissue probability maps was over $0.5 .^{41,42}$

\section{Construction of KNE96 template}

We constructed the KNE96 template, composed of high resolution 3D structural MR images from 96 Korean normal elderly subjects, using the DARTEL toolbox in SPM8 $8{ }^{43}$ First, each of MRI scans was segmented using the segmentation routine in SPM8, and then the spatial transformation files derived from the segmentation routine was used to import the MRI scan into the rigidly transformed tissue probability maps of WM, GM and CSF. Following this, the tissue types were simultaneously registered (i.e., WM with WM, GM with GM, and CSF with CSF). The initial template is constructed as the mean of these $96 \mathrm{MRI}$ images in the construction group. Deformations from this template to each of the individual images were computed and then the template was updated by applying the inverse deformations to the images and averaging the deformed images. This procedure was repeated based on multi-level pyramid approach, beginning with a more coarse registration then ending with the more detailed registration. After a total of 36 iterations, three probability maps of the KNE96 template were finally constructed. Finally, for T1 image of the KNE96 template, we warped individual T1 images using the flow fields that store deformation information and created the mean of the 96 warped T1 images.

In addition, we also constructed two gender-specific templates (KNE48M template for male and KNE48F template for female) by dividing the subjects in the construction group into two subgroups by gender and repeating the template construction process for 48 male subjects and 48 female subjects, respectively.

\section{Validation of the KNE96 template}

To validate the KNE96 template, we used a nonlinear version of the ICBM152 atlas (i.e., ICBM152 template) as a comparison template. ${ }^{15,16}$ We compared the deformations that resulted from image registration using the KNE96 and ICBM 152 templates. Each MRI scan in the validation group was registered to both the KNE96 and ICBM152 templates using DARTEL ${ }^{43}$ We used displacement vectors and Jacobian determinants to analyze the deformation of each subject to each template. ${ }^{44}$ For each voxel, the Euclidean distance between the subject and its deformed image was obtained as the magnitude of the displacement vector, $\overrightarrow{d v}$. Furthermore, the Jacobian determinant is an indicator of volumetric change at each voxel under deformation. Its value is $>1,1$, or $<1$ if there is volume expansion, preservation, or contraction, respectively. Therefore, we derived two kinds of maps for each test subject registered to each template: the magnitude of displacement vectors (i.e., mag-displacement) and log of Jacobian determinants (i.e., log-Jacobian).

We analyzed the differences in the mag-displacement and log-Jacobian maps between the KNE96 and ICBM152 templates using voxel-based morphometry (VBM). Because the mag-displacement and log-Jacobian maps were generated in each template space that was used as the reference for registration, these maps were spatially normalized so that the coordinates were in MNI space, using the DARTEL toolbox. ${ }^{43}$ Comparison between the KNE96- and ICBM152-registered mag-displacement and log-Jacobian maps was performed 
with a paired-samples t-test for each voxel. With Bonferroni correction, $\mathrm{p}<6.9 \times 10^{-9}$ was considered a statistically significant difference.

In addition to VBM analysis of the mag-displacement and log-Jacobian maps, to explore the region-specific differences between the KNE96 and ICBM152 templates, we determined the deformation for each anatomical region of the Talairach atlas. ${ }^{11,45}$ First, voxels were classified into 1105 anatomical regions using the Talairach atlas. Second, for each Talairach re- gion, the significant averaged differences (SADs) of the magdisplacement and the log-Jacobian were computed as follows:

$$
\begin{aligned}
& \operatorname{SAD}_{\text {mag-displacement }}\left(R_{t}\right)=R_{\text {mag-displacement }}\left(R_{t}\right) \times A D_{\text {mag-displa }} \\
& \operatorname{SAD}_{\text {log-Jacobian }}\left(R_{t}\right)=R_{\text {log-Jacobian }}\left(R_{t}\right) \times A D_{\text {log-Jacobian }}\left(R_{t}\right)
\end{aligned}
$$

with

$$
A D_{\text {mag-displacement }}\left(R_{t}\right)=\frac{1}{N_{S}} \frac{1}{N_{R_{t}}} \sum_{j=1}^{N_{S}} \sum_{x_{i} \in R_{t}}\left\|\overrightarrow{d v}_{S_{j}, \text { ICBM152 }}\left(x_{i}\right)|-| \overrightarrow{d v}_{S_{i}, \text { KNE96 }}\left(x_{i}\right)\right\|
$$

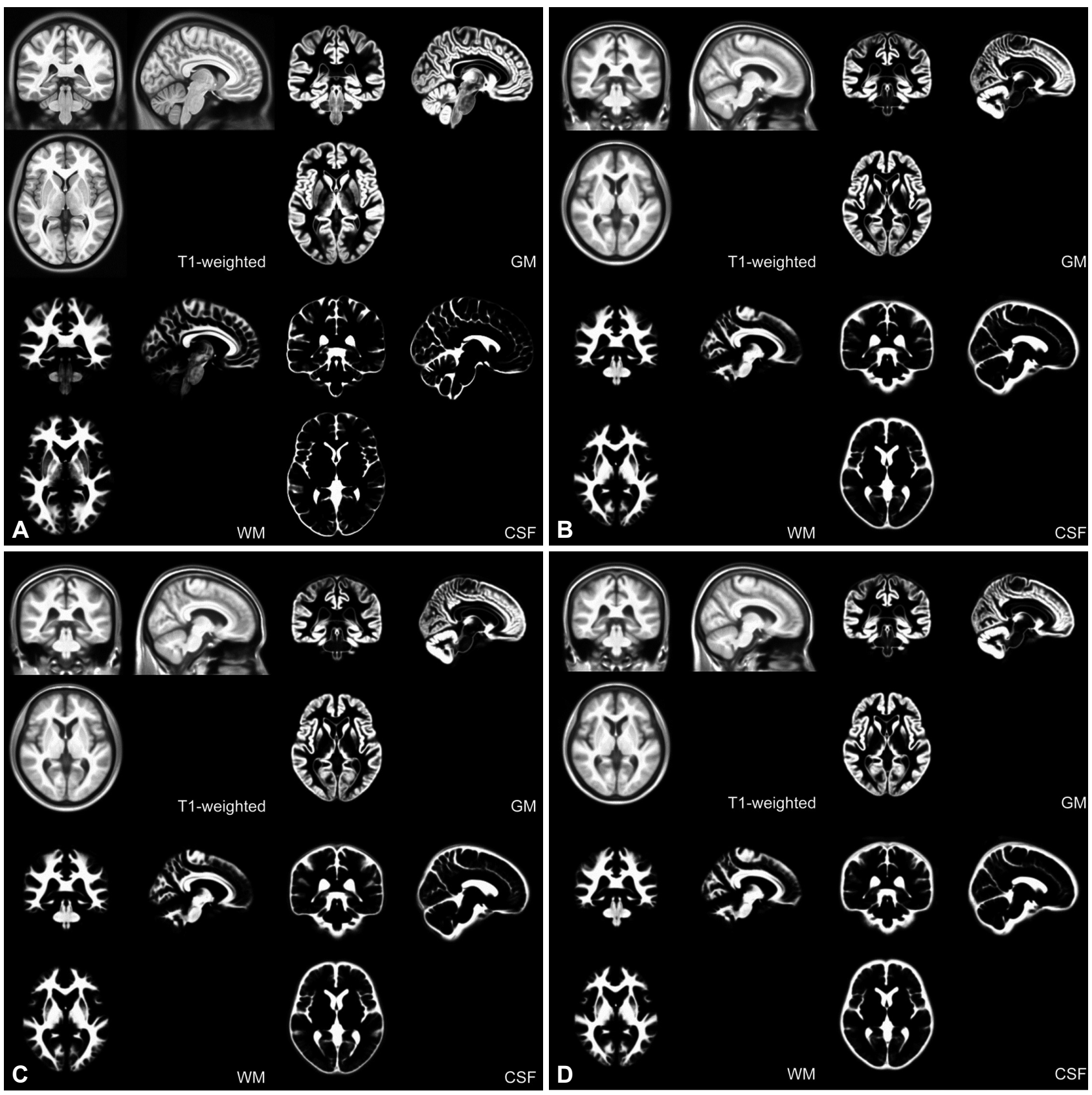

Figure 1. Brain MRI templates. A: ICBM152 template. B: KNE96 template. C: KNE48M template. D: KNE48F template. GM: gray matter, WM: white matter, CSF: cerebrospinal fluid, ICBM: the international consortium for brain mapping, KNE: Korean normal elderly brain template. 


$$
A D_{\text {log-Jacobian }}\left(R_{t}\right)=\frac{1}{N_{S}} \frac{1}{N_{R_{t}}} \sum_{j=1}^{N_{S}} \sum_{x_{i} \in R_{t}}\left|\log _{2} J_{S_{j}, \text { ICBMI52 }}\left(x_{i}\right)-\log _{2} J_{S_{j}, \text { KNE96 }}\left(x_{i}\right)\right|
$$

Here, $x_{i}$ represents the $i$ th voxel in the mag-displacement and log-Jacobian maps and $N_{R_{t}}$ represents the number of voxels that belong to the tth Talairach region, $R_{t} . S_{j}$ and $N_{s}=48$ represent the $j$ th subject and the number of subjects in the validation group, respectively. Significant voxel rates, $R_{\text {mag-dis- }}$ placemen $\left(R_{t}\right)$ and $R_{\text {log-jacobian }}\left(R_{t}\right)$, were defined as the ratio of the number of significant voxels from the paired-samples t-tests to the total number of voxels in the Talairach region, $R_{t}$.

\section{RESULTS}

\section{Global brain features}

Figure 1 shows the KNE96, KNE48M, KNE48F, and IC BM152 templates. In Korean subjects, male brains were longer, wider, taller, and thus larger than the female brains. However, the ratios of $\mathrm{W} / \mathrm{L}, \mathrm{H} / \mathrm{L}$, and $\mathrm{H} / \mathrm{W}$ were comparable between male and female brains (Table 2). The KNE96 template was shorter but wider than the ICBM152 template (Table 3), which was consistent with previous observations that report differences in global hemispheric shape and size between Occidental and Oriental brains. ${ }^{29,30,46}$

\section{Comparison of registration-induced image deformation between the KNE96 and ICBM152 templates}

Figure 2 shows the differences in the mag-displacement and log-Jacobian maps between the KNE96 and ICBM152 templates. As shown in Figure 2A, we found that the mag-displacement and log-Jacobian induced by registration to the KNE96 template were much smaller than those induced by registration to the ICBM152 template in most brain regions. Figure 2B illustrates the significant voxels of the VBM analysis overlaid on corresponding standard T1 image. This indicated that there is less deformation and a lower risk of volumetric errors if KNE96 is used as the reference template in neuroimaging studies in the Korean elderly population.

Figure 3 shows the SAD maps of the mag-displacement and the log-Jacobian. The mag-displacement SAD map shows large values in some Talairach regions within the frontal and occipital lobes while the log-Jacobian SAD map shows large values in the frontal and occipital lobes, and the thalamus.

By considering each Talairach region as a data point, we constructed a two-dimensional scatter plot in which the $y$-axis is the mag-displacement SAD and the $\mathrm{x}$-axis is the log-Jacobian SAD, as shown in Figure 4. Because we excluded small Talairach regions that had a volume less than $500 \mathrm{~mm}^{3}$, Figure 4 contains 355 data points. And then, we performed a twostep cluster analysis in PASW Statistics 18.0 (IBM SPSS Inc,

Table 2. Measurement of global brain features of Korean normal elderly subjects in the construction group

\begin{tabular}{lcccr}
\hline & Korean elderly & Male & Female & p-value \\
\hline Length & $160.0 \pm 7.00$ & $161.4 \pm 6.89$ & $158.6 \pm 6.88$ & 0.016 \\
Width & $137.7 \pm 6.31$ & $140.4 \pm 6.17$ & $135.0 \pm 5.21$ & $<0.001$ \\
Height & $118.5 \pm 4.73$ & $120.3 \pm 4.37$ & $116.8 \pm 4.46$ & $<0.001$ \\
W/L & $0.862 \pm 0.05$ & $0.872 \pm 0.06$ & $0.853 \pm 0.05$ & 0.053 \\
H/L & $0.741 \pm 0.03$ & $0.746 \pm 0.04$ & $0.737 \pm 0.03$ & 0.111 \\
H/W & $0.861 \pm 0.04$ & $0.858 \pm 0.04$ & $0.866 \pm 0.04$ & 0.251 \\
Volume $\left(10^{4}\right)$ & $161.4 \pm 19.2$ & $166.0 \pm 18.2$ & $156.8 \pm 19.2$ & 0.004 \\
\hline
\end{tabular}

W/L: width to length, H/L: height to length, H/W: height to width

Table 3. Measurement of global brain features of four templates

\begin{tabular}{lcccc}
\hline & KNE96 & KNE48M & KNE48F & ICBM152 \\
\hline Length & 160 & 161 & 158 & 135 \\
Width & 136 & 137 & 114 & 134 \\
Height & 115 & 117 & 0.85 & 124 \\
W/L & 0.85 & 0.85 & 0.72 & 0.77 \\
H/L & 0.72 & 0.73 & 0.84 & 0.72 \\
H/W & 0.85 & 0.85 & 149.7 & 0.93 \\
Volume $\left(10^{4}\right)$ & 153.3 & 156.8 & 156.9 \\
\hline
\end{tabular}

KNE: Korean normal elderly brain template, ICBM: The International Consortium for Brain Mapping, W/L: width to length, H/L: height to length, H/W: height to width 
2009, Chicago, IL, USA) to partition these data points relative to their mag-displacement and log-Jacobian SAD values. Tables 4 and 5 list the Talairach regions with large mag-dis- placement and log-Jacobian SADs, respectively. Volume change indicates the ratio of the deformed volume using the ICBM152 template to that using the KNE96 template. The
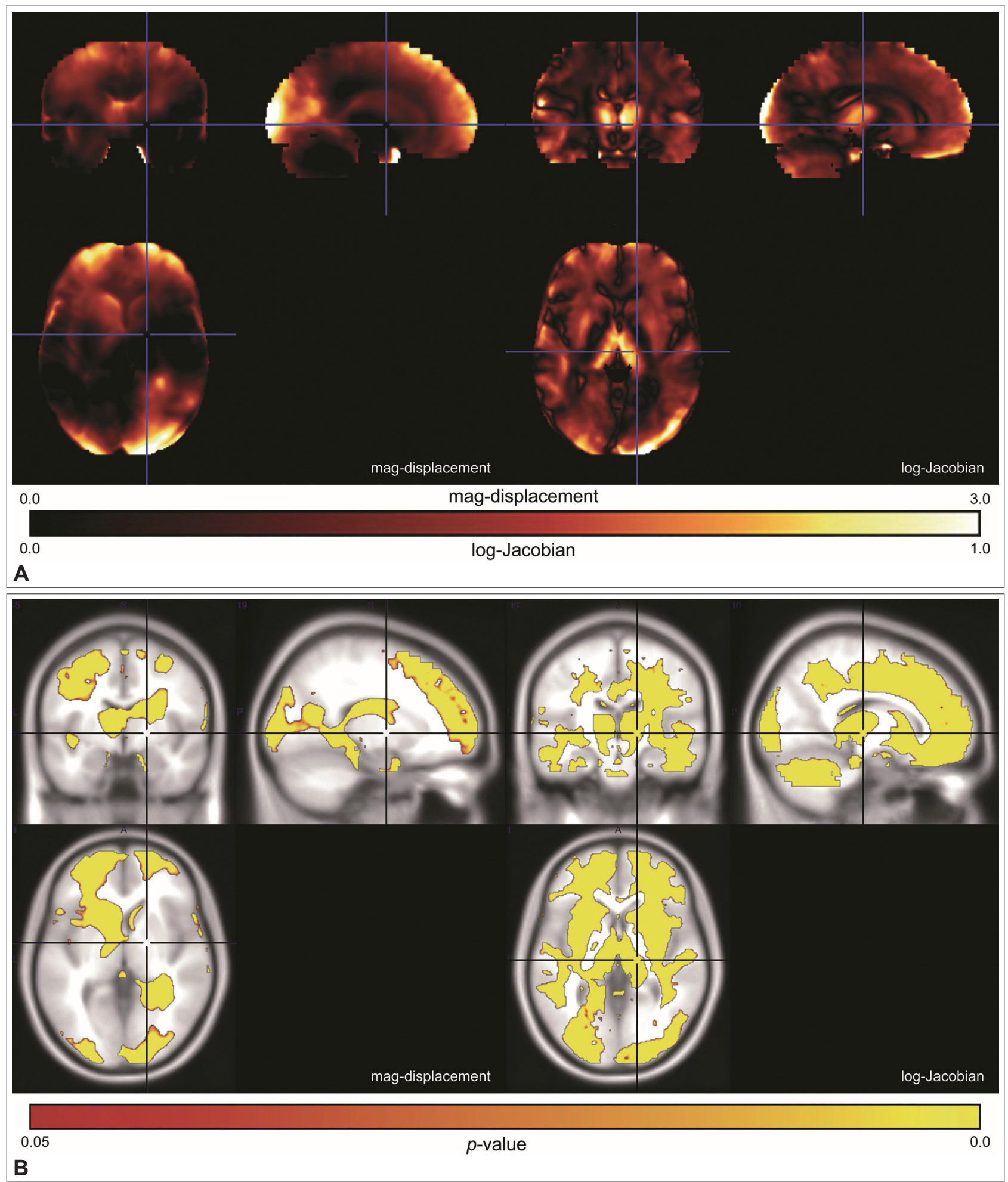

Figure 2. Comparison of registration-induced image deformation between the KNE96 and ICBM152 templates. A: Difference maps of the mag-displacement and log-Jacobian. B: Significant voxels of the voxel-based morphometry (VBM) analysis of the mag-displacement and log-Jacobian. KNE: Korean normal elderly brain template, ICBM: the international consortium for brain mapping. 


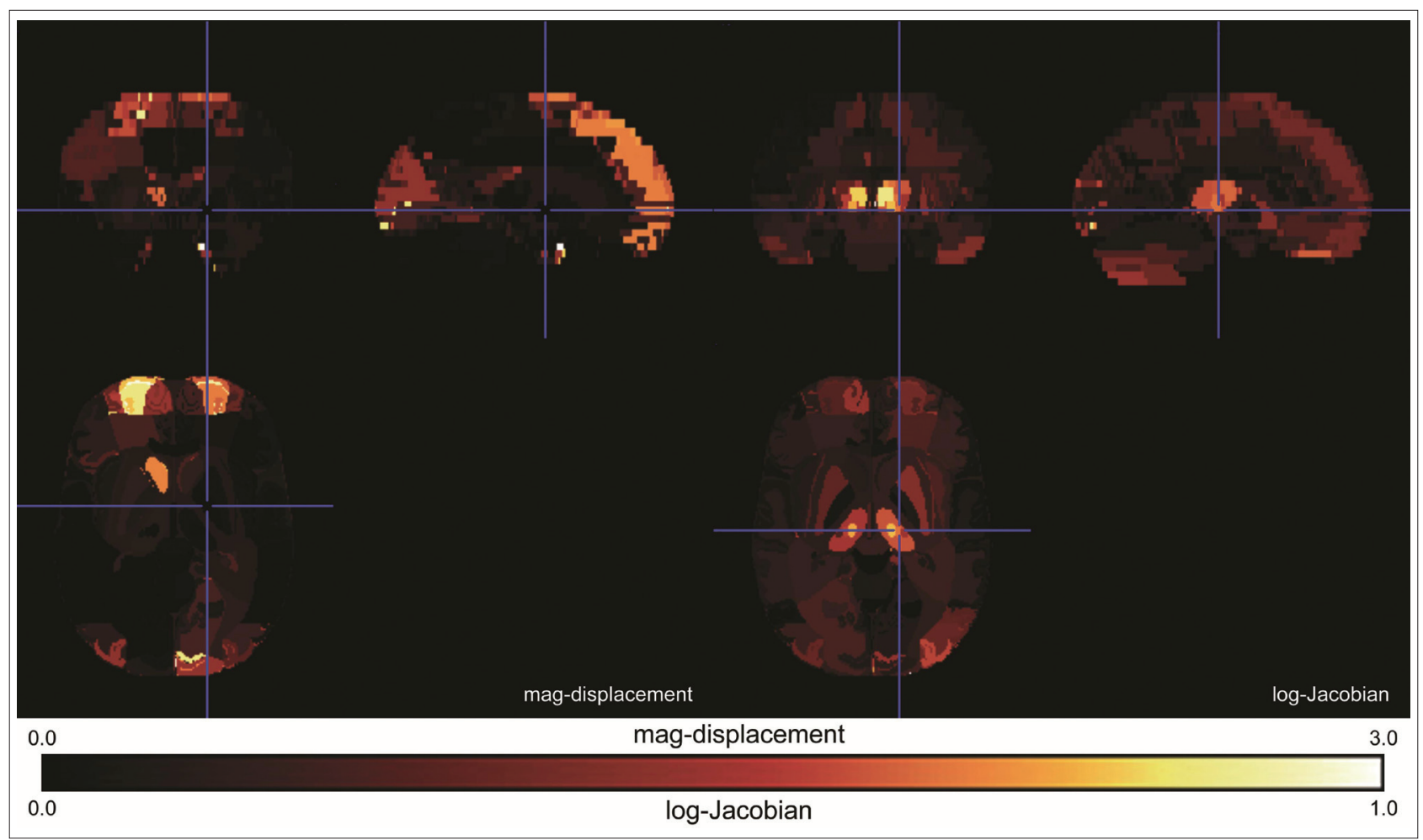

Figure 3. Comparison of significant averaged difference (SAD) maps of the mag-displacement and log-Jacobian between the KNE96 and ICBM152 templates. KNE: Korean normal elderly brain template, ICBM: the international consortium for brain mapping.

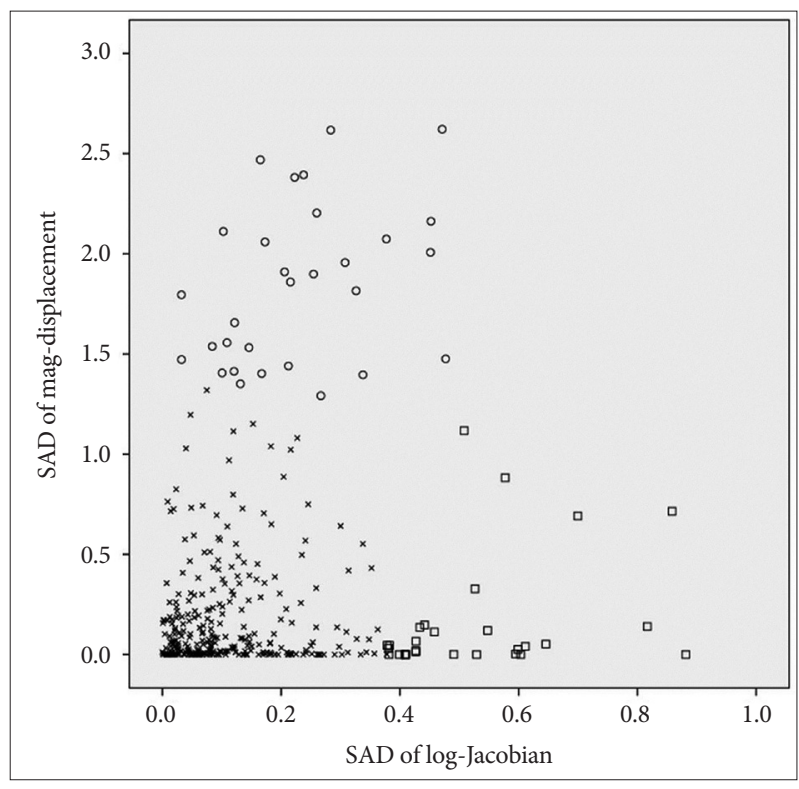

Figure 4. Significant averaged differences (SADs) distribution of 355 Talairach regions with volume larger than $500 \mathrm{~mm}^{3}$.

Talairach regions with large mag-displacement SAD values (circles in Figure 4) include the superior, medial, and middle frontal gyrus in the frontal lobe, lingual gyrus and cuneus in the occipital lobe, and caudate in the sub-lobar (Table 4). The Talairach regions with large log-Jacobian SAD values (boxes in Figure 4) include the thalamus in the sub-lobar, superior, middle, and inferior occipital gyrus in the occipital lobe, rectal gyrus in the frontal lobe, inferior semilunar lobule in the posterior lobe, and fusiform gyrus in the temporal lobe (Table 5). These regions may be subject to considerably larger changes in position or volume if the ICBM152 was used instead of the KNE96 as a reference template for neuroimaging studies in elderly Koreans.

\section{DISCUSSION}

Although many volumetric brain MRI studies have been based on Caucasian populations, the number of studies using Asian brain MRI images is steadily rising. Figure 5 shows the number of articles containing the words 'brain', 'MRI', and either 'Asian', 'Korean', 'Chinese', or 'Japanese' published in NeuroImage for the period of 2005 to 2014. Thus, the construction and validation of a group-specific template using Asian subjects is a necessary alternative to the public atlases currently provided with many research tools.

In this study, we constructed a new Korean normal elderly brain template using high resolution 3.0T T1 structural MR images of 96 Korean elderly subjects $(\mathrm{M} / \mathrm{F}=48 / 48)$ aged 60 years or older $(\mathrm{M}=69.5 \pm 6.2$ years, $\mathrm{F}=70.1 \pm 7.0$ years $)$. We validated this template by comparing the magnitude of regis- 
tration-induced deformation between the KNE96 and ICBM 152 templates using 48 Korean elderly subjects $(\mathrm{M} / \mathrm{F}=24 / 24)$ aged 60 years or older ( $\mathrm{M}=71.5 \pm 5.9$ years, $\mathrm{F}=72.8 \pm 5.1$ years). Although this study focused on the construction and validation of a Korean normal elderly brain template, it has demonstrated that further research into other phenotypic characteristics, such as gender and disease condition, should be taken into account for optimal and powerful analyses of regional brain morphometry.

In computational brain MRI studies, spatial normalization is necessary to compare structural changes between individual brains. During spatial normalization, brains are transformed to a standard template space, which intrinsically changes the position and local volume at every voxel in the image. In order to quantify the deformation, we used the magnitude of displacement vectors (i.e., mag-displacement) and the log of the Jacobian determinants (i.e., log-Jacobian). Using these two deformation measurements, we compared the image registration of Korean elderly brains to the KNE96 template and to the ICBM152 template. In particular, we explored re-

Table 4. Thirty Talairach regions with large significant averaged differences (SADs) of mag-displacement

\begin{tabular}{|c|c|c|c|c|c|c|c|}
\hline \multicolumn{5}{|c|}{ Talairach labels } & \multicolumn{3}{|c|}{ SADs } \\
\hline Hemisphere & Lobe & Gyrus & Tissue & Cell & Mag-displacement (mm) & Log-Jacobian & Volume change (\%) \\
\hline $\mathrm{RC}$ & $\mathrm{OL}$ & LG & GM & BA17 & 2.622 & 0.471 & 138.6 \\
\hline $\mathrm{LC}$ & $\mathrm{FL}$ & SFG & GM & BA8 & 2.469 & 0.165 & 112.1 \\
\hline LC & FL & MeFG & GM & BA8 & 2.394 & 0.238 & 117.9 \\
\hline LC & FL & SFG & GM & BA10 & 2.382 & 0.223 & 116.7 \\
\hline $\mathrm{RC}$ & FL & SFG & GM & BA8 & 2.204 & 0.260 & 119.7 \\
\hline $\mathrm{LC}$ & FL & MeFG & GM & BA11 & 2.162 & 0.452 & 136.8 \\
\hline LC & SL & Cau & GM & $\mathrm{CauH}$ & 2.112 & 0.103 & 107.4 \\
\hline $\mathrm{RC}$ & FL & SFG & GM & BA9 & 2.059 & 0.173 & 112.7 \\
\hline LC & FL & MFG & GM & BA11 & 2.007 & 0.451 & 136.7 \\
\hline LC & FL & SFG & GM & BA11 & 1.956 & 0.308 & 123.8 \\
\hline LC & FL & SFG & GM & BA9 & 1.910 & 0.206 & 115.3 \\
\hline $\mathrm{RC}$ & FL & SFG & GM & BA10 & 1.899 & 0.254 & 119.3 \\
\hline $\mathrm{RC}$ & FL & MeFG & GM & BA8 & 1.860 & 0.216 & 116.1 \\
\hline $\mathrm{RC}$ & FL & SFG & GM & BA6 & 1.815 & 0.326 & 125.4 \\
\hline LC & FL & MFG & GM & BA6 & 1.796 & 0.032 & 102.2 \\
\hline $\mathrm{RC}$ & FL & $\mathrm{MeFG}$ & GM & BA9 & 1.557 & 0.109 & 107.8 \\
\hline LC & SL & Cau & GM & CauB & 1.538 & 0.084 & 106.0 \\
\hline LC & FL & SFG & GM & BA6 & 1.532 & 0.146 & 110.6 \\
\hline LC & FL & $\mathrm{MeFG}$ & GM & BA10 & 1.476 & 0.477 & 139.2 \\
\hline $\mathrm{RC}$ & SL & $\mathrm{Cau}$ & GM & $\mathrm{CauB}$ & 1.472 & 0.032 & 102.3 \\
\hline $\mathrm{RC}$ & $\mathrm{OL}$ & Cun & GM & BA18 & 1.440 & 0.212 & 115.8 \\
\hline $\mathrm{RC}$ & $\mathrm{OL}$ & Cun & GM & BA17 & 1.414 & 0.121 & 108.7 \\
\hline $\mathrm{RC}$ & $\mathrm{LL}$ & Unc & GM & BA28 & 1.403 & 0.167 & 112.3 \\
\hline LC & $\mathrm{OL}$ & MOG & GM & BA18 & 1.396 & 0.338 & 126.4 \\
\hline LC & FL & SFG & WM & $*$ & 2.617 & 0.283 & 121.7 \\
\hline $\mathrm{RC}$ & FL & SFG & WM & $*$ & 2.074 & 0.377 & 129.9 \\
\hline LC & FL & MFG & WM & $*$ & 1.656 & 0.122 & 108.8 \\
\hline $\mathrm{RC}$ & $\mathrm{OL}$ & Cun & WM & $*$ & 1.406 & 0.100 & 107.2 \\
\hline LC & FL & $\mathrm{MeFG}$ & WM & $*$ & 1.292 & 0.267 & 120.3 \\
\hline $\mathrm{RC}$ & FL & SFG & $*$ & $*$ & 1.351 & 0.131 & 109.5 \\
\hline
\end{tabular}

LC: left cerebrum, RC: right cerebrum, FL: frantal lobe, LL: limbic lobe, OL: occipital lobe, SL: sub-lobar, LG: lingual gyrus, SFG: superior frontal gyrus, MeFG: medial frontal gyrus, MFG: middle frontal gyrus, MOG: middle occipital gyrus, Cau: caudate, Cun: cuneus, Unc: uncus, GM: gray matter, WM: white matter, BA: brodmann area, CauB: caudate body, CauH: caudate head 
gion-specific differences in mag-displacement and log-Jacobian maps between the KNE96 template and the ICBM152 template. In this study, we have presented these region-specific differences for each anatomical region defined by the Talairach atlas ${ }^{11,45}$ and reported the Talairach regions with large SAD values.

We found that regions in the frontal and occipital lobe, and thalamus show a large positional or local volume change when Korean elderly subjects were registered to the ICBM152 template compared to when registered to the KNE96 tem- plate. In addition, our measurements of the global brain features of Korean elderly subjects reflect previously reported differences in global shape and size between Occidental and Oriental brains. ${ }^{29,30,46}$ Therefore, computational neuroimaging studies that measure, compare, and interpret the anatomical features of these regions in brains from Asian elderly populations using public atlases that are based on Caucasian populations, such as ICBM152 template, may have large measurement errors and thus false study results.

For example, we calculated the log-Jacobian SAD in the me-

Table 5. Twenty-nine Talairach regions with large significant averaged differences (SADs) of log-Jacobian

\begin{tabular}{|c|c|c|c|c|c|c|c|}
\hline \multicolumn{5}{|c|}{ Talairach labels } & \multicolumn{3}{|c|}{ SADs } \\
\hline Hemisphere & Lobe & Gyrus & Tissue & Cell & Mag-displacement (mm) & Log-Jacobian & Volume change (\%) \\
\hline $\mathrm{RC}$ & SL & Th & GM & $\mathrm{MDN}$ & 0.000 & 0.882 & 184.2 \\
\hline $\mathrm{RC}$ & $\mathrm{OL}$ & SOG & GM & BA19 & 0.715 & 0.858 & 181.3 \\
\hline $\mathrm{LC}$ & SL & Th & GM & $\mathrm{MDN}$ & 0.140 & 0.817 & 176.1 \\
\hline $\mathrm{RC}$ & SL & Th & GM & VLN & 0.052 & 0.646 & 156.5 \\
\hline $\mathrm{RC}$ & SL & Th & GM & $*$ & 0.041 & 0.611 & 152.8 \\
\hline $\mathrm{RC}$ & SL & Th & GM & Pul & 0.026 & 0.599 & 151.4 \\
\hline LC & FL & RG & GM & BA11 & 0.003 & 0.595 & 151.0 \\
\hline $\mathrm{RC}$ & $\mathrm{OL}$ & MOG & GM & BA18 & 0.883 & 0.577 & 149.2 \\
\hline $\mathrm{RC}$ & $\mathrm{OL}$ & IOG & GM & BA18 & 0.120 & 0.548 & 146.2 \\
\hline LC & SL & Th & GM & $*$ & 0.328 & 0.526 & 144.0 \\
\hline LC & SL & Th & GM & VLN & 1.118 & 0.508 & 142.2 \\
\hline $\mathrm{RC}$ & PL & ISL & GM & $*$ & 0.001 & 0.491 & 130.4 \\
\hline $\mathrm{RC}$ & FL & MFG & GM & BA11 & 0.113 & 0.458 & 137.4 \\
\hline $\mathrm{LCl}$ & PL & ISL & GM & $*$ & 0.136 & 0.434 & 135.1 \\
\hline $\mathrm{RC}$ & SL & LN & GM & Put & 0.066 & 0.427 & 134.4 \\
\hline $\mathrm{RC}$ & $\mathrm{TL}$ & FG & GM & BA20 & 0.019 & 0.427 & 134.4 \\
\hline $\mathrm{RC}$ & $\mathrm{TL}$ & ITG & GM & BA20 & 0.000 & 0.410 & 132.8 \\
\hline $\mathrm{LC}$ & SL & Th & GM & Pul & 0.000 & 0.409 & 132.7 \\
\hline LC & LL & CG & GM & BA23 & 0.000 & 0.399 & 131.9 \\
\hline $\mathrm{LCl}$ & PL & Pyr & GM & $*$ & 0.046 & 0.383 & 130.4 \\
\hline $\mathrm{RC}$ & FL & RG & GM & BA11 & 0.000 & 0.382 & 130.3 \\
\hline $\mathrm{RC}$ & $\mathrm{TL}$ & FG & GM & BA37 & 0.046 & 0.379 & 130.0 \\
\hline $\mathrm{RC}$ & $\mathrm{OL}$ & IOG & WM & $*$ & 0.692 & 0.700 & 162.4 \\
\hline LC & FL & RG & WM & $*$ & 0.000 & 0.603 & 144.3 \\
\hline $\mathrm{RC}$ & $\mathrm{FL}$ & $\mathrm{RG}$ & WM & $*$ & 0.000 & 0.529 & 151.9 \\
\hline LC & $\mathrm{OL}$ & IOG & WM & $*$ & 0.148 & 0.442 & 135.8 \\
\hline $\mathrm{RC}$ & $\mathrm{TL}$ & FG & WM & $*$ & 0.013 & 0.427 & 134.4 \\
\hline $\mathrm{RC}$ & $\mathrm{TL}$ & ITG & WM & $*$ & 0.000 & 0.410 & 132.9 \\
\hline $\mathrm{RC}$ & $\mathrm{TL}$ & FG & $*$ & $*$ & 0.031 & 0.381 & 130.2 \\
\hline
\end{tabular}

LC: left cerebrum, RC: right cerebrum, LCl: left cerebellum, FL: frontal lobe, LL: Limbic lobe, OL: occipital lobe, PL: posterior lobe, TL: temporal lobe, SL: sub-lobar, MFG: middle frontal gyrus, IOG: inferior occipital gyrus, MOG: middle occipital gyrus, SOG: superior occipital gyrus, ITG: inferior temporal gyrus, CG: cingulate gyrus, FG: fusiform gyrus, RG: rectal gyrus, ISL: inferior semilunar lobule, LN: lentiform nucleus, Th: thalamus, Pyr: pyramis, GM: gray matter, WM: white matter, BA: brodmann area, MDN: medial dorsal nucleus, VLN: ventral lateral nucleus, Pul: pulvinar, Put: putamen 


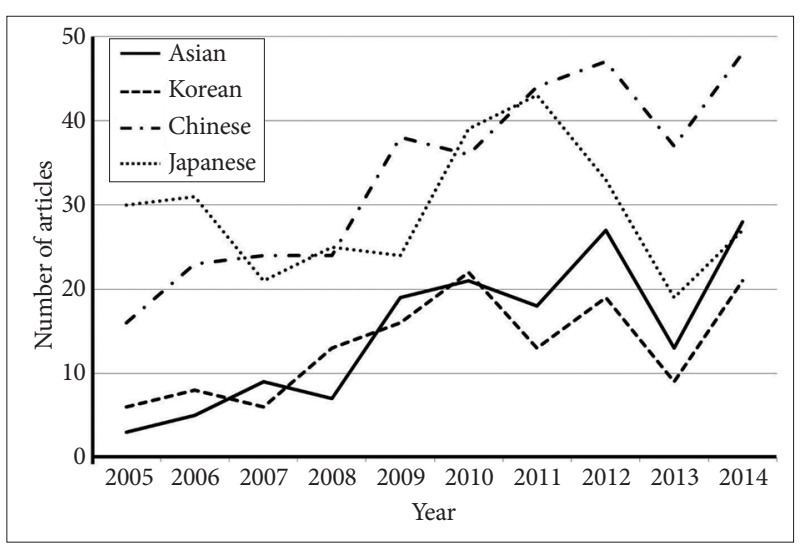

Figure 5. The number of articles containing the Asian-related words published in the Neurolmage.

dial dorsal nucleus of the thalamus in the right cerebrum to be 0.88 . This means that if Korean elderly subjects are registered to the ICBM152 template, this region might be 1.84 times larger when compared with registration to the KNE96 template. Some volumetric studies have reported bilateral thalamus volume reduction in Alzheimer's disease (AD) patients when compared with normal control subjects. ${ }^{47-52}$ The thalamus is known to be involved in directing attention and suppressing irrelevant sensory inputs, ${ }^{53}$ in particular the medial dorsal nucleus is important for memory functions. ${ }^{54}$ However, in these studies, MRI images from Caucasian elderly subjects were transformed to the ICBM152 template and the thalamus was segmented. The use of the ICBM152 template as a reference atlas for Asian elderly subjects may substantially increase the risk of volumetric errors and therefore exaggerate or diminish thalamus volume reduction in $\mathrm{AD}$ patients. To investigate the consistency of the studies among different ethnic groups, it is important to use a populationspecific template, such as the KNE96 template, for spatial normalization and segmentation of brain images from Asian populations.

Tables 4 and 5 show the asymmetry of deformation in the cuneus of the occipital lobe and fusiform gyrus of the temporal lobe. These regions have large mag-displacement or log-Jacobian SAD values in the right hemisphere, which are not present in the left hemisphere. Although the cause of this asymmetry is unclear, the results demonstrate that the right cuneus and fusiform gyrus had a greater positional or volumetric change when registered to the ICBM152 template than the left cuneus and fusiform gyrus. For example, in the fusiform gyrus of the temporal lobe, there was an approximately $130 \%$ volume change in the right hemisphere when compared with $115 \%$ volume change in the left hemisphere. Therefore, the use of the ICBM152 template as a reference atlas leads to unreliable results and the KNE96 template is strongly recommended in the Korean elderly population.
The KNE96 template was derived from 96 Korean normal elderly subjects, more than 60 years old, whereas the ICBM152 template was made base on 152 normal young age-Caucasian subjects. Therefore, unfortunately, the difference between the KNE96 template and the ICBM152 template includes not only the racial difference but also the age difference. In this study, we limited our scope to construct and validate the KNE96 template that would be fundamental for brain MRI studies about aging and geriatric of Korean elderly population, rather than to investigate the differences in brain structures from two groups having different age and race.

The KNE96, KNE48M, and KNE48F templates are available to download from http://recode.webnode.kr/news/knekorean-normal-elderly-group-template/.

\section{Acknowledgments}

This work was partially supported by a grant from the National Research Foundation of Korea (NRF) funded by the Korea government (MSIP) (No. 2010-0028631) and by a grant from the Korean Health Technology R\&D Project, Ministry of Health and Welfare, Republic of Korea [grant number HI09C1379(A092077)]. This work also was supported by grants from Esai Korea (No.06-2011-173 and No. 06-2009-041).

\section{REFERENCES}

1. Giedd JN, Blumenthal J, Jeffries NO, Castellanos FX, Liu H, Zijdenbos A, et al. Brain development during childhood and adolescence: a longitudinal MRI study. Nat Neurosci 1999;2:861-863.

2. Ashburner J, Friston KJ. Voxel-based morphometry -- the methods. Neuroimage 2000;11:805-821.

3. Kasai K, Iwanami A, Yamasue H, Kuroki N, Nakagome K, Fukuda M. Neuroanatomy and neurophysiology in schizophrenia. Neurosci Res 2002;43:93-110

4. Jack CR Jr, Bernstein MA, Fox NC, Thompson P, Alexander G, Harvey $D$, et al. The Alzheimer's Disease Neuroimaging Initiative (ADNI): MRI methods. J Magn Reson Imaging 2008;27:685-691.

5. Collins DL, Neelin P, Peters TM, Evans AC. Automatic 3D intersubject registration of MR volumetric data in standardized Talairach space. J Comput Assist Tomogr 1994;18:192-205.

6. Ashburner J, Friston KJ. Nonlinear spatial normalization using basis functions. Hum Brain Mapp 1999;7:254-266.

7. Lancaster JL, Fox PT, Downs H, Nickerson DS, Hander TA, El Mallah $\mathrm{M}$, et al. Global spatial normalization of human brain using convex hulls. J Nucl Med 1999;40:942-955.

8. Mazziotta J, Toga A, Evans A, Fox P, Lancaster J, Zilles K, et al. A probabilistic atlas and reference system for the human brain: International Consortium for Brain Mapping (ICBM). Philos Trans R Soc Lond B Biol Sci 2001;356:1293-1322.

9. Toga AW, Thompson PM. Maps of the brain. Anat Rec 2001;256:37-53.

10. Fox PT, Perlmutter JS, Raichle ME. A stereotactic method of anatomical localization for positron emission tomography. J Comput Assist Tomogr 1985;9:141-153.

11. Talairach J, Tournoux P. Co-Planar Stereotaxic Atlas of the Human Brain: 3-D Proportional System - An Approach to Cerebral Imaging. New York: Thieme Medical Publishers; 1988.

12. Chau W, McIntosh AR. The Talairach coordinate of a point in the MNI space: how to interpret it. Neuroimage 2005;25:408-416.

13. Ahsan RL, Allom R, Gousias IS, Habib H, Turkheimer FE, Free S, et al. Volumes, spatial extents and a probabilistic atlas of the human basal ganglia and thalamus. Neuroimage 2007;38:261-270.

14. Shattuck DW, Mirza M, Adisetiyo V, Hojatkashani C, Salamon G, Narr 
$\mathrm{KL}$, et al. Construction of a 3D probabilistic atlas of human cortical structures. Neuroimage 2008;39:1064-1080.

15. Evans AC, Janke AL, Collins DL, Baillet S. Brain templates and atlases. Neuroimage 2012;62:911-922.

16. Fonov V, Evans AC, Botteron K, Almli CR, McKinstry RC, Collins DL, et al. Unbiased average age-appropriate atlases for pediatric studies. Neuroimage 2011;54:313-327.

17. Statistical Parametric Mapping (SPM). Available at: http://www.fil.ion. ucl.ac.uk/spm/. Accessed September 18, 2015.

18. MRI Studio. Available at: http://www.mristudio.org/. Accessed September 18, 2015.

19. FMRIB Software Library (FSL). Available at: http://fsl.fmrib.ox.ac.uk/ fsl/fslwiki/. Accessed September 18, 2015.

20. Jenkinson M, Beckmann CF, Behrens TEJ, Woolrich MW, Smith SM. FSL. Neuroimage 2012;62:782-790.

21. Jackson FL. Race and ethnicity as biological constructs. Ethn Dis 1992; 2:120-125.

22. Buckner RL, Head D, Parker J, Fotenos AF, Marcus D, Morris JC, et al. A unified approach for morphometric and functional data analysis in young, old, and demented adults using automated atlas-based head size normalization: reliability and validation against manual measurement of total intracranial volume. Neuroimage 2004;23:724-738.

23. Smith CD, Chebrolu H, Wekstein DR, Schmitt FA, Markesbery WR. Age and gender effects on human brain anatomy: a voxel-based morphometric study in healthy elderly. Neurobiol Aging 2007;28:1075-1087.

24. Moriguchi Y, Ohnishi T, Kawachi T, Mori T, Hirakata M, Yamada M, et al. Specific brain activation in Japanese and Caucasian people to fearful faces. Neuroreport 2005;16:133-136.

25. Altaye M, Holland SK, Wilke M, Gaser C. Infant brain probability templates for MRI segmentation and normalization. Neuroimage 2008; 43:721-730.

26. Wilke M, Holland SK, Altaye M, Gaser C. Template-O-Matic: a toolbox for creating customized pediatric templates. Neuroimage 2008; 41:903-913.

27. Chee MW, Chen KH, Zheng H, Chan KP, Isaac V, Sim SK, et al. Cognitive function and brain structure correlations in healthy elderly East Asians. Neuroimage 2009;46:257-269.

28. Kochunov P, Fox PC, Lancaster J, Tan LH, Amunts K, Zilles K, et al. Localized morphological brain differences between English-speaking Caucasians and Chinese-speaking Asians: new evidence of anatomical plasticity. Neuroreport 2003;14:961-964.

29. Lee JS, Lee DS, Kim J, Kim YK, Kang E, Kang H, et al. Development of Korean standard brain templates. J Korean Med Sci 2005;20:483-488.

30. Tang Y, Hojatkashani C, Dinov ID, Sun B, Fan L, Lin X, et al. The construction of a Chinese MRI brain atlas: a morphometric comparison study between Chinese and Caucasian cohorts. Neuroimage 2010;51:3341.

31. Sato K, Taki Y, Fukuda H, Kawashima R. Neuroanatomical database of normal Japanese brains. Neural Netw 2003;16:1301-1310.

32. Uchiyama HT, Seki A, Tanaka D, Koeda T, Jcs group. A study of the standard brain in Japanese children: morphological comparison with the MNI template. Brain Dev 2013;35:228-235.

33. Kim TH, Park JH, Lee JJ, Jhoo JH, Kim BJ, Kim JL, et al. Overview of the Korean Longitudinal Study on Cognitive Aging and Dementia. Alzheimers Dement 2013;9(Suppl 4):626-627.

34. Hughes CP, Berg L, Danziger WL, Coben L Martin RL. A new clinical scale for the staging of dementia. Br J Psychiatry 1982;140:566-572.

35. American Psychiatric Association. Diagnostic and Statistical Manual of Mental Disorders: DSM-IV. Task Force on DSM-IV. Washington,
DC: American Psychiatric Association; 1994.

36. Lee JH, Lee KU, Lee DY, Kim KW, Jhoo JH, Kim JH, et al. Development of the Korean version of the Consortium to Establish a Registry for Alzheimer's Disease Assessment Packet (CERAD-K): Clinical and neuropsychological assessment batteries. J Gerontol B Psychol Sci Soc Sci 2002;57:P47-P53.

37. Oldfield RC. The assessment and analysis of handedness: the Edinburgh inventory. Neuropsychologia 1971;9:97-113.

38. BrainSuite. Available at: http://neuroimage.usc.edu/neuro/BrainSuite/. Accessed September 18, 2015.

39. Shattuck DW, Leahy RM. BrainSuite: an automated cortical surface identification tool. Med Image Anal 2002;6:129-142.

40. Ashburner J, Friston KJ. Unified segmentation. Neuroimage 2005;26: 839-851.

41. Colliot O, Chételat G, Chupin M, Desgranges B, Magnin B, Benali H, et al. Discrimination between Alzheimer disease, mild cognitive impairment, and normal aging by using automated segmentation of the hippocampus. Radiology 2008;248:194-201.

42. Chen Y, Wolk DA, Reddin JS, Korczykowski M, Martinez PM, Musiek ES, et al. Voxel-level comparison of arterial spin-labeled perfusion MRI and FDG-PET in Alzheimer disease. Neurology 2011;77:1977-1985.

43. Ashburner J. A fast diffeomorphic image registration algorithm. Neuroimage 2007;38:95-113.

44. Zhang Y, Zhang J, Hsu J, Oishi K, Faria AV, Albert M, et al. Evaluation of group-specific, whole-brain atlas generation using Volume-based Template Estimation (VTE): application to normal and Alzheimer's populations. Neuroimage 2014;84:406-419.

45. Talairach. Available at: http://www.talairach.org/. Accessed September 18, 2015.

46. Zilles K, Kawashima R, Dabringhaus A, Fukuda H, Schormann T. Hemispheric shape of European and Japanese brains: 3-D MRI analysis of intersubject variability, ethnical, and gender differences. Neuroimage 2001;13:262-271.

47. Karas GB, Scheltens P, Rombouts SA, Visser PJ, van Schijndel R, Fox $\mathrm{NC}$, et al. Global and local gray matter loss in mild cognitive impairment and Alzheimer's disease. Neuroimage 2004;23:708-716.

48. Chételat G, Landeau B, Eustache F, Mézenge F, Viader F, de la Sayette $\mathrm{V}$, et al. Using voxelbased morphometry to map the structural changes associated with rapid conversion in MCI: a longitudinal MRI study. Neuroimage 2005;27:934-946.

49. Teipel SJ, Born C, Ewers M, Bokde AL, Reiser MF, Möller HJ, et al. Multivariate deformation-based analysis of brain atrophy to predict Alzheimer's disease in mild cognitive impairment. Neuroimage 2007; 38:13-24.

50. de Jong LW, van der Hiele K, Veer IM, Houwing JJ, Westendorp RG, Bollen EL, et al. Strongly reduced volumes of putamen and thalamus in Alzheimer's disease: an MRI study. Brain 2008;131:3277-3285.

51. Cherubini A, Péran P, Spoletini I, Di Paola M, Di Iulio F, Hagberg GE, et al. Combined volumetry and DTI in subcortical structures of mild cognitive impairment and Alzheimer's disease patients. J Alzheimer Dis 2010;19:1273-1282.

52. Roh JH, Qiu A, Seo SW, Soon HW, Kim JH, Kim GH, et al. Volume reduction in subcortical regions according to severity of Alzheimer's disease. J Neurol 2011;258:1013-1020.

53. Newman J. Thalamic contributions to attention and consciousness. Conscious Cogn 1995;4:172-193.

54. Van der Werf YD, Witter MP, Uylings HB, Jolles J. Neuropsychology of infarctions in the thalamus: a review. Neuropsychologia 2000;38:613627. 